\title{
Development of a machine vision system for a real time precision sprayer
}

\author{
Jérémie Bossu*, Christelle Gée* and Frédéric Truchetet ${ }^{+}$ \\ * ENESAD/DSI, UP GAP, 21 Bld Olivier de Serres, Quétigny, France \\ + UMR 5158 uB-CNRS, 12 rue de la Fonderie, Le Creusot, France
}

Received $26^{\text {th }}$ May 2008; accepted $26^{\text {th }}$ November 2008

\begin{abstract}
In the context of precision agriculture, we have developed a machine vision system for a real time precision sprayer. From a monochrome CCD camera located in front of the tractor, the discrimination between crop and weeds is obtained with image processing based on spatial information using a Gabor filter.This method allows to detect the periodic signals from the non-periodic ones, and enables us to enhance the crop rows, whereas weeds have a patchy distribution. Thus, weed patches were clearly identified by a blob-coloring method. Finally, we use a pinhole model to transform the weed patch coordinates image in world coordinates in order to activate the right electro-pneumatic valve of the sprayer at the right moment.
\end{abstract}

Keywords: Gabor filter, image processing, precision agriculture, weeds, crop, spraying.

\section{Introduction}

In the year 1980 in the USA, an agriculture called precision agriculture appeared with the development of the new technologies such as GPS, remote sensors... It is usually defined as "the right dose, at the right place and at the right moment". The purpose of the precision agriculture is to reduced chemical inputs, which have an environmental and economic impact. The reduction of chemical inputs can be applied according to the following two approaches:

- Mapping concept,

- Real-time concept.

Sensors can be embedded in agricultural engines[1] or aircraft[2,3] in order to provide useful informations on the heterogeneities of the soil, crop and weeds. Particular attention can be paid to the reduction of herbicides, which are the main pollutants in agriculture. In the past, our laboratory developed a multispectral imaging system embedded in a small aircraft[2] in order to realize a weed infestation map after flying over crop fields. At the same time, we study the development of a machine vision system for a real time precision sprayer using a camera embedded in a tractor in order to spray specifically on plant infested-areas. Herbicides saving

Correspondence to: <c.gee@enesad.fr $>$

Recommended for acceptance by $<$ D.Fofi and R.Seulin $>$

ELCVIA ISSN:1577-5097

Published by Computer Vision Center / Universitat Autònoma de Barcelona, Barcelona, Spain 
can be done by developing various systems in real time for site specific spraying to the infested areas. These systems use the optical sensors (photodiodes) and are able to discriminate plants and soil by their reflectance. The most famous ones are Weedseeker[4], Detectspray[5] and Sprayvision [6]. However, these systems cannot discriminate between crop and weeds. More recently, Åstrand et al. [7, 8] have developed a robot, with two vision systems to guide it through the crop rows, whose aim is to remove weeds in the inter-row with a mechanical tool. However, this method of detection is limited to some crops (salad, sugar beet, etc...), where seedling is done with the drilling method. The aim of this paper is to present the development of a real time precision sprayer based on machine vision, devoted to the inter-row weed detection in cereal crop fields, from a spatial approach in order to target herbicide spraying.

\section{Materials and methods}

\subsection{Experimental set-up}

The figure, Fig.1, shows an overview of the experimental set-up, a camera, tractor and a sprayer where an electro-pneumatic valve has been placed in front of each nozzle

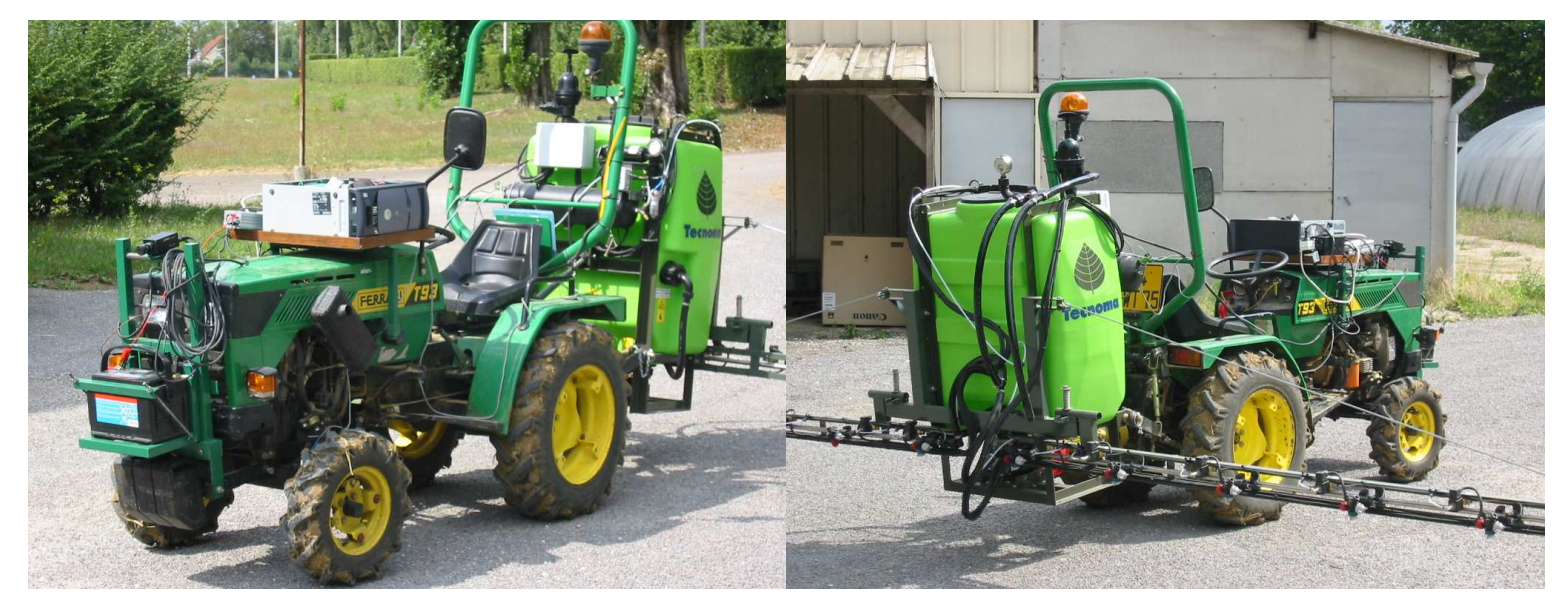

Figure 1: Overview of the precision sprayer.

\subsubsection{The precision sprayer}

The Tecnoma "TS200" sprayer is composed of a six meter boom with twelve nozzles spaced by fifty centimeters. The hydraulic circuit was similar to a conventional sprayer with an output from the main pump fed to a pressure control valve (a constant pressure regulation). In the context of precision agriculture, two sensors have been embedded on the tractor: a vision system placed in front of the tractor and a speed sensor fixed on a front wheel. Moreover, the sprayer has been modified (Fig.2): each nozzle can be turned on or off separately from a control unit (called a spray control system) via the electro-pneumatic valve (EPV). The Spray Control System (SCS) is based on the use of a microcontroller (PIC 16C765 from Microchip) linked to a computer via a serial port. During the herbicide applications, this system receives the weed locations via the computer. The positions are defined after image processing of the acquired image. The SCS allows the EPV to be turned on/off separately, depending on the tractor speed, when herbicide is required. A specific pneumatic circuit (compressor) has been developed in order to maintain a sufficient pressure (4 bars) for good behavior of the EPV. 


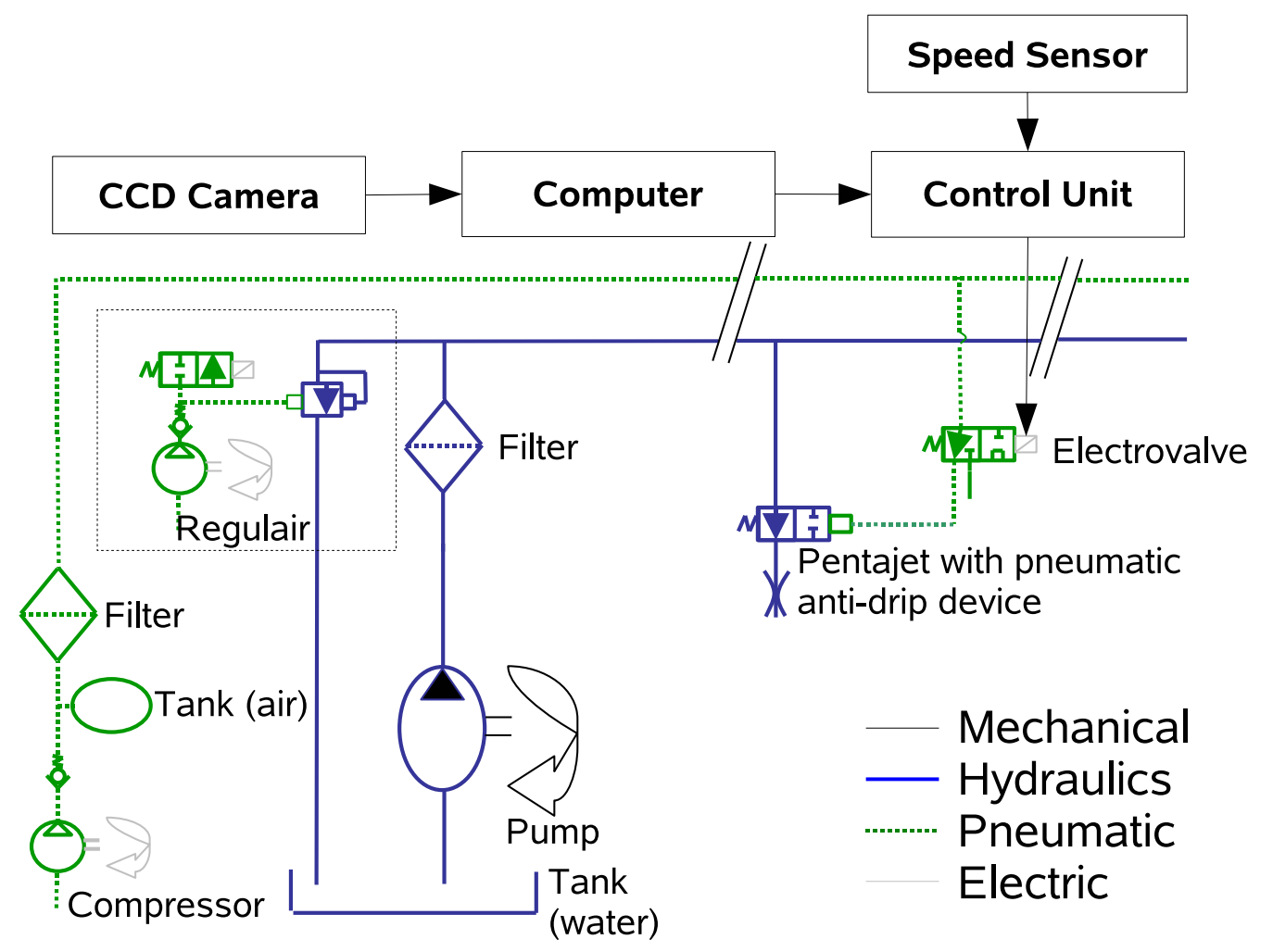

Figure 2: General flowchart of the precision sprayer

\subsubsection{Agronomic scene}

At the present time, the first trials are done in a car park of the institute ENESAD where we simulated an agronomic scene. Based on the fact that soil is grey, we created crop rows composed of a white stripe pattern (made with adhesive) in order to model crop seedlings as observed on Fig.3.a. The average bandwidth of a row is fixed at five centimeters, and the space between two consecutive rows is about sixteen centimeters (simulation of a cereal field). Weeds have been made with white paper in different forms and randomly placed in inter-row of the crop.
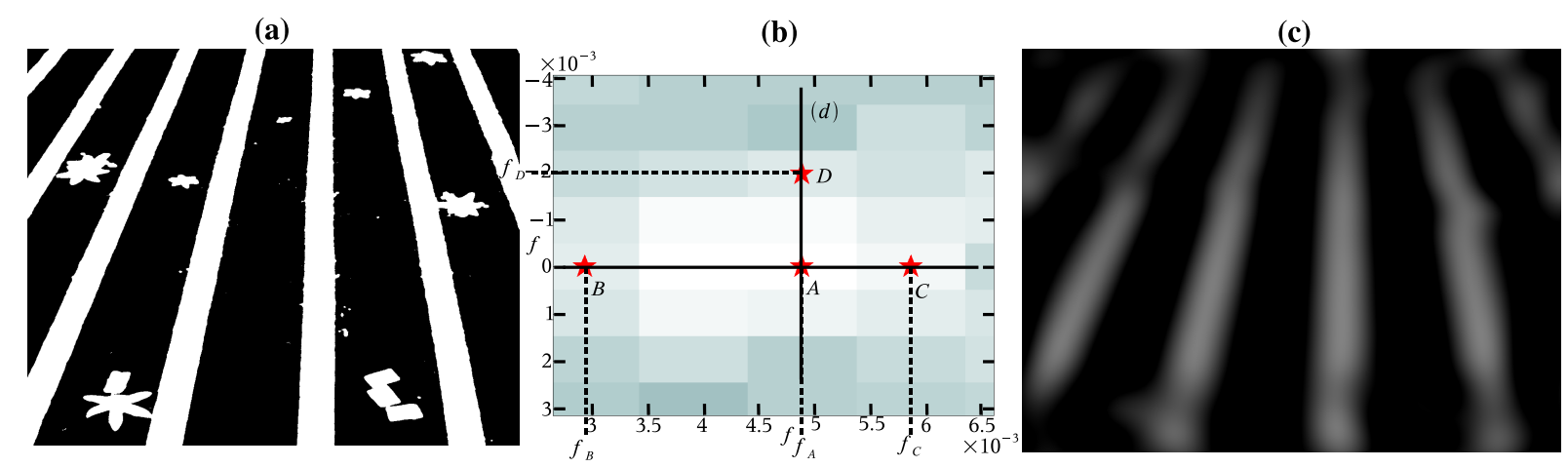

Figure 3: (a) Simulation of a cereal field (bandwidth $=5 \mathrm{~cm}$ and row spacing $=16 \mathrm{~cm}$ ) in the presence of weeds localized in the inter-row. (b) Zoom of the Fourier transform of the simulated image. (c) Result of Gabor filtering 


\subsubsection{Images acquisition}

Images are acquired by a monochrome CCD camera (Sony U1000, $1598 \times 1199$ ) located in front of the tractor and inclined with a $58^{\circ}$ tilt-angle. According to perspective effects, the real dimensions of the agronomic scene are estimated to be $2.44 \times 1.45 \mathrm{~m}$. The camera is connected to an on-board computer by a National Instruments Imaq PCI/PXI-1428 frame grabber, and the computer used an Intel Celeron processor with $2.4 \mathrm{GHz}$ frequency with $256 \mathrm{MB}$ of RAM. For real-time applications, the image processing is done with the software Microsoft Visual C++ using OpenCV (Open Source Computer Vision)[9] and IPP (Integrated Performance Primitive) developed by Intel Software[10].

\subsection{Method : image processing}

In order to test the robustness of the discrimination algorithm, we have used simulated images. It is a very useful tool for evaluating the accuracy of any algorithms under various conditions with a perfect knowledge of every initial parameters of the natural scene (weed and crop pixel, weed infestation rate). Moreover, it is possible to simulate different types of natural scenes which are sometimes difficult to find in the surrounding of the laboratory and in a given space of time. A set of agronomic images has been created with a simulation engine based on a spatial plant growth model developed by Jones et al.[11]. First, the virtual field(Fig.6.a), considered as a black and white two dimensional surface, is created by a periodic sowing pattern for crop plants and the punctual and patchy distributions of weed plants are modelled by two different stochastic process (Poisson process and Neymann-Scott process)[12]. A discrete statistical analysis has been developed assuming that the weed spatial distribution is a random process with no memory between successive events (two built images) and that occurrence of the emergences of weed plants compare to crop plants in field is very low. In this model, the initial inter-row weed infestation rate is a parameter and it is defined as:

$$
\text { initial WIR } \text { inter-row }=\frac{\text { inter-row weed pixels } \times 100}{(\text { crop }+ \text { inter-row weed }) \text { pixels }}
$$

The initial crop rate is defined by:

$$
\text { initial } C R=100-\text { initial WIR } \text { inter-row }
$$

Secondly, a virtual camera with pre-defined intrinsic (CCD-height: Hccd $=5.28 \mathrm{~mm}$ and CCD-width: Lccd=7mm; focal lens: $\mathrm{f}=8.5 \mathrm{~mm}$ ) and extrinsic parameters (camera tilt-angle $=58^{\circ}$, camera pan-angle $=0^{\circ}$; camera swingangle $=0^{\circ}$; camera Height $=1.05 \mathrm{~m}$ ) is located in the field. From the pinhole camera model (appendix A, we are able to map the real world coordinates of a point into its pixel coordinates in the image space. Thus, a virtual image (in grey levels) can be obtained as illustrated in Figure 1.

\subsubsection{Gabor filtering}

Presentation of the Gabor filter To detect crop rows in image, we use a spatial method based on the Gabor filter[2].

The bi-dimensional Gabor filter[13, 14, 15] is derived from a mono-dimensional Gabor filter[16]. It is defined as a modulation of a gaussian function by a complex oscillator. The general form is defined by:

$$
g(x, y)=\frac{1}{\pi \sigma_{x} \sigma_{y}} e^{-\left[\frac{x^{2}}{2 \sigma_{x}^{2}}+\frac{y^{2}}{2 \sigma_{y}^{2}}\right]} e^{j 2 \pi\left(u_{0} x+v_{0} y\right)}
$$

As the crop rows are coarsely vertically oriented, we prefer a filter following the horizontal direction to distinguish between the periodic signals from the non-periodic signals along this direction. Thus, we have a real filter following a direction, and the previous equation becomes:

$$
g(x, y)=\frac{1}{\pi \sigma_{x} \sigma_{y}} e^{-\left[\frac{x^{2}}{2 \sigma_{x}^{2}}+\frac{y^{2}}{2 \sigma_{y}^{2}}\right]} \cos \left(2 \pi u_{0} x\right)
$$


We can separate this function into a product of two filter functions such as:

$$
\begin{array}{lllllll}
g(x, y) & =e^{-\frac{x^{2}}{2 \sigma_{x}^{2}}} \cos \left(2 \pi u_{0} x\right) & \times & e^{-\frac{y^{2}}{2 \sigma_{y}^{2}}} & & \times & \frac{1}{\pi \sigma_{x} \sigma_{y}} \\
g(x, y) & = & m(x) & \times & h(y) & \times & N
\end{array}
$$

The part $m(x)$ (eq.5) represents a monodimensional Gabor filter centered on $u_{0}$ frequency along the horizontal direction with a standard deviation $\sigma_{x}$. This filter can be a band pass or a low pass filter following $\sigma_{x}$ value. If $\sigma_{x}$ value is low, the bandwidth in the frequency domain is high and the filter becomes a low pass. Otherwise, the bandwidth in the frequency domain is low and the filter is a band pass. To preserve a band pass behavior, $\sigma_{x}$ must increase for small value of $u_{0}$. In the frequency domain, the standard deviation is equal to $\frac{1}{2 \pi \sigma_{x}}$.

The part, $h(y)$, is a gaussian function with a standard deviation $\sigma_{y}$ orthogonal to the horizontal direction. In the frequency domain, the standard deviation is $\frac{1}{2 \pi \sigma_{y}}$. It is a low pass filter.

The part, $N$, is a coefficient allowing an unit gain.

Its Fourier transform is given by:

$$
G(u, v)=e^{-2 \pi^{2} \sigma_{y}^{2} v^{2}}\left[e^{-2 \pi^{2} \sigma_{x}^{2}\left(u-u_{0}\right)^{2}}+e^{-2 \pi^{2} \sigma_{x}^{2}\left(u+u_{0}\right)^{2}}\right]
$$

Fourier transform and detection of parameters of Gabor filter We perform a Fourier transform on the image acquired (image in grey level) in order to detect the parameters of the Gabor filter:

- The central frequency $u_{0}$

- The standard deviation $\sigma_{x}$ along horizontal direction

- The standard deviation $\sigma_{y}$ along vertical direction

To extract the parameter $u_{0}$, we work on the half frequency space because the Fourier transform is symmetric. We search for the maximum level of magnitude denoted by $A$. This maximum corresponds to the main frequency component present in the original image. This is situated along the horizontal frequency axis, and we denote the frequency associated by $f_{A}$. It is the central frequency of the filter:

$$
u_{0}=f_{A}
$$

Standard deviations along the two directions, vertical and horizontal are difficult to determine. So we perform an algorithm based on the magnitude level[17]. We search for three other levels, denoted by $B, C$ and $D$, depending on the level of $A$.

As we use a normalized Fourier transform, the maximum of the module is equal to $1 \mathrm{~dB}$. The $B$ and $C$ levels are located along the horizontal frequency axis. We define $B$ level at about $89 \%$ of the level of $A$, so $B \simeq 0.89 \mathrm{~dB}$, and $C$ level is about $87 \%$ of $A$ so $C \simeq 0.87 \mathrm{~dB}$. The $D$ level is along the straight line $(d)$, which is orthogonal to the straight line $(B C)$. We search on $(d)$, where the level $D$ is about $83 \%$ of $A$ so $D=0.83$ $\mathrm{dB}$.

We denote by $f_{B}, f_{C}$ and $f_{D}$ the $f_{x}$ and $f_{y}$ coordinates associated to levels $B, C$ and the $D$ level respectively (Fig.3.b). The difference between the frequencies $f_{C}$ and $f_{B}$ allows us to find the standard deviation $\sigma_{x}$ along the horizontal direction. The frequency $f_{D}$ is used to define $\sigma_{y}$ and the standard deviation along the vertical direction. The standard deviations are given by:

$$
\begin{aligned}
\sigma_{x} & =\frac{1}{\pi\left(\left|f_{C}\right|-\left|f_{B}\right|\right)} \\
\sigma_{y} & =\frac{1}{2 \pi\left|f_{D}\right|}
\end{aligned}
$$


This entire process and the associated parameters have been defined after an extensive experimental study on numerous simulated agronomic images. This method is done for images with a low perspective effect.

The convolution between this filter and the original image allows us to enhance the crop rows. The result of the filtering is shown Fig.3.c.

\subsubsection{Discrimination between crop and weeds}

After the crop row detection with a Gabor filter, we must differentiate between crop and weeds. The image is then binarized with a threshold equal to the average value of the intensity of the pixels composing the image. Consequently, all vegetation pixels are white in color, whereas the black color represents soil pixels (this image is noted $a$ ). A threshold is also applied with the filtering image (noted $b$ ). Afterwards we use the logical function $A N D$ between these two images in order to obtain the crop map (notes $c$ )(Fig.4.a, white color), so:

$$
c=a \cdot b
$$

Then with the logical function $X O R$ between the previous result and the initial image, we are able to deduce a weed infestation map (noted $w$ ) as shown in Fig.4.a, where weeds are in black:

$$
w=a \oplus c
$$

According to the equations 10 and 11, we can demonstrate that:

$$
w=a \cdot \bar{b}
$$

(a)

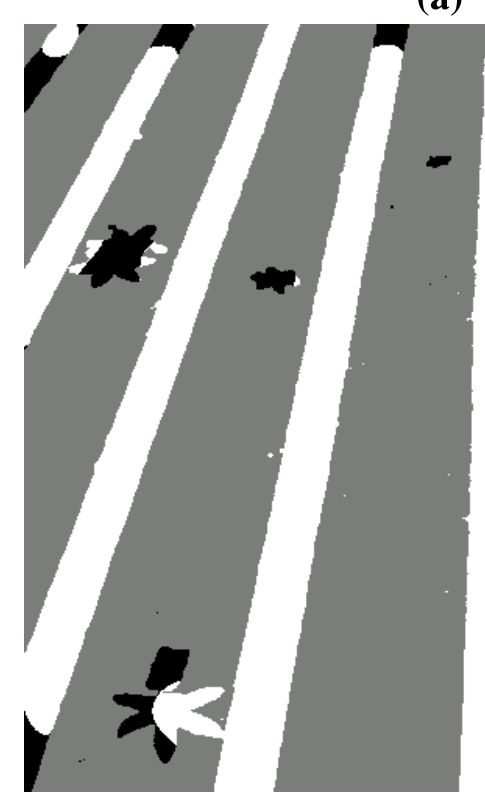

(b)
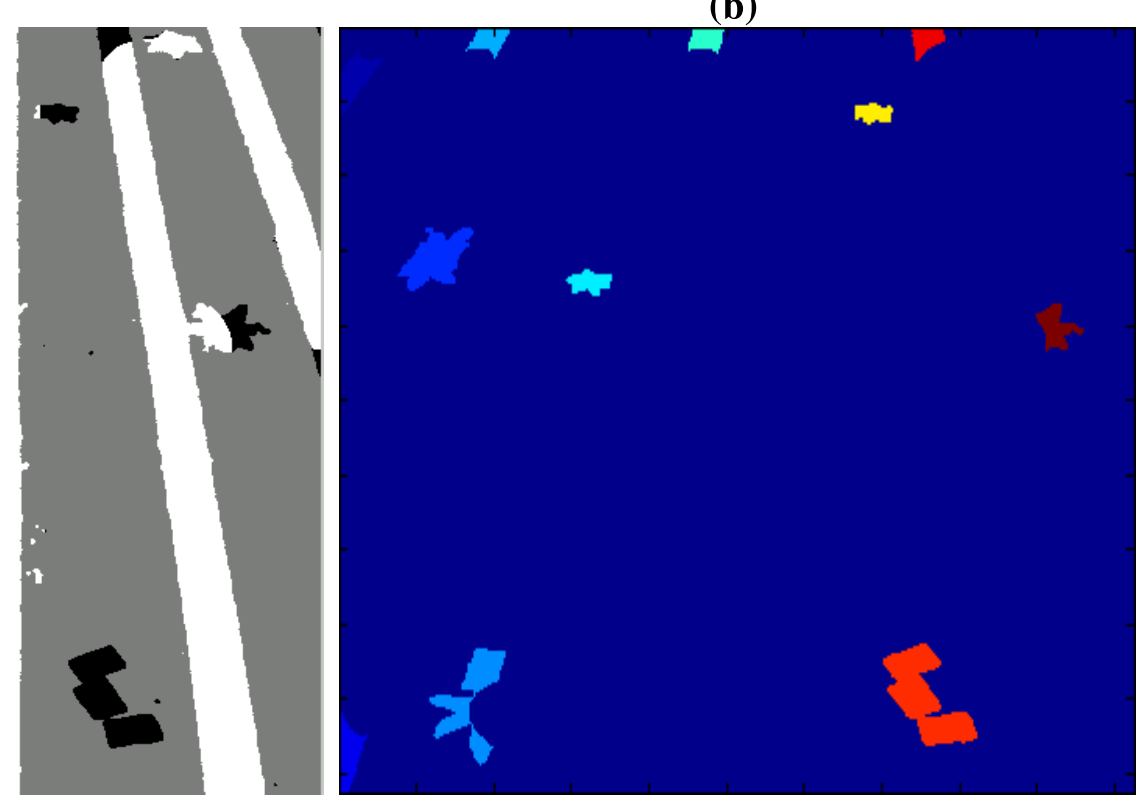

Figure 4: (a)Discrimination between crop (white) and weeds (black), soil is grey. (b)The inter-row weed infestation map segmented by a blob-coloring method.

\subsubsection{Infestation map}

From the crop/weed discrimination (Fig.4.a), we are able to create a weed infestation map. From this map, a region based segmentation is done in order to group weed pixels into patches. To carry on this treatment, 
we use a blob-coloring method[18, 19]. Applying the inverse pinhole model, it is possible to determine the coordinates of these regions in the real world depending on the intrinsic and extrinsic parameters of the optical system shown in table 1 . The details of the coordinate transformation can be found in appendix A.

\begin{tabular}{|l|l|}
\hline Intrinsic & Extrinsic \\
\hline $\mathrm{f}=8.5 \mathrm{~mm}$ & $\mathrm{H}=1.05 \mathrm{~m}$ \\
\hline $\mathrm{dx}=\mathrm{dy}=4,4 \mu \mathrm{m}$ & $\phi=58^{\circ}$ \\
\hline
\end{tabular}

Table 1: Intrinsic and extrinsic parameters values of the optical system.

According to the size of these regions, a decision is made on whether to conserve theme or not. Indeed, if the size of a patch in the real world is inferior to the minimal size of the seedling $(4 \mathrm{~cm} \times 2 \mathrm{~cm})$, we remove this patch. The figure, Fig.4.b, shows a map where all weed patches have been selected.

\subsubsection{EPV choice}

From the weed infestation map, each EPV can be controlled independently. The figure, Fig.5.a, shows a schematic view of the tractor with the spray boom. On this figure, we can see the origin of the real world $\left(x_{w}, y_{w}\right)$, which is located in the middle of the spray boom along the direction $x_{w}$. For each weed patch, only two extrema coordinates along the $x$ axis have been selected and are denoted $x_{w \min }$ and $x_{w \max }$. The average of these coordinates for each of these patches allows us is to assign the right nozzle to each weed patch. Lastly, taking into account the tractor velocity, the opening and the closing of the valves are defined by the maximal and minimal values of the coordinates of the weed patches.

(a)

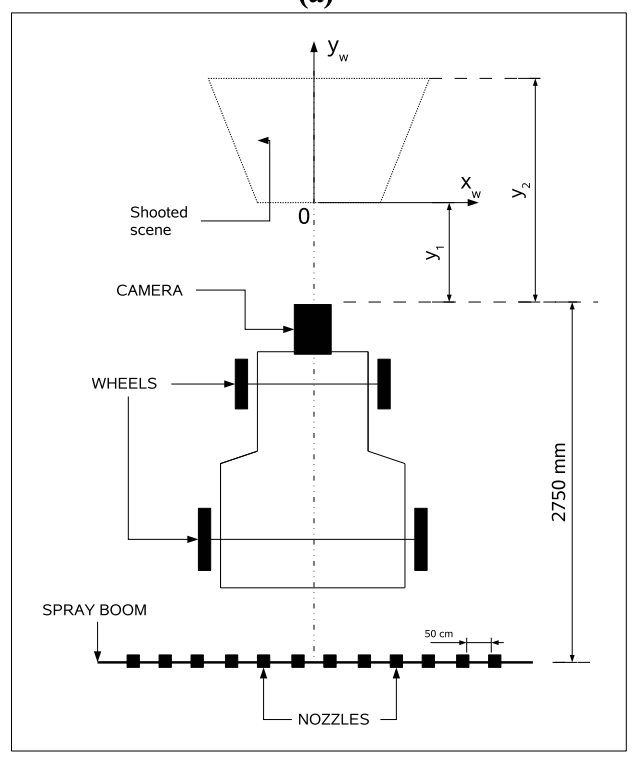

(b)

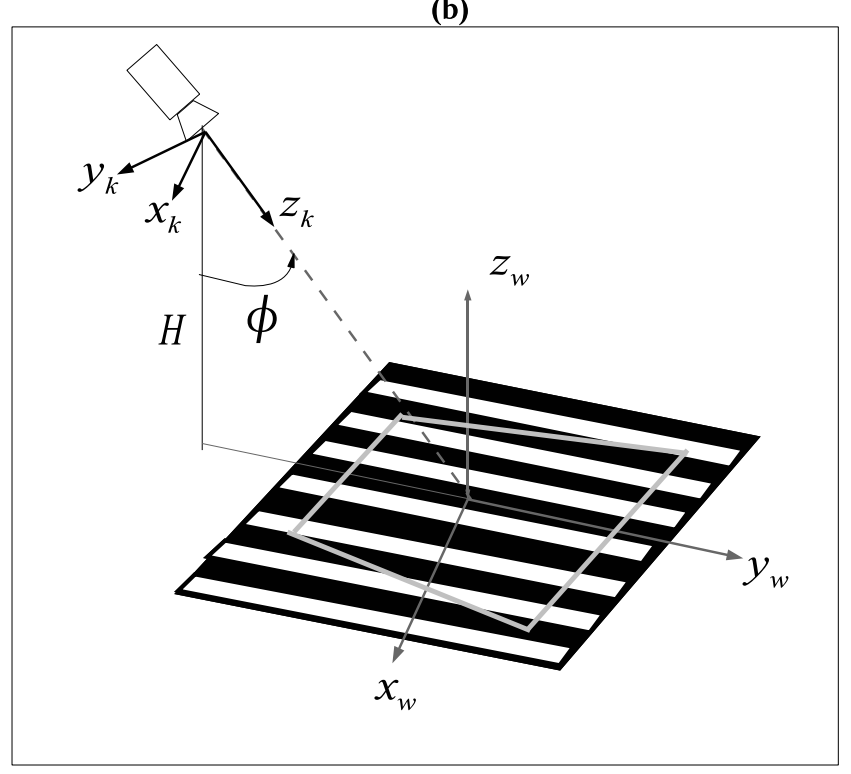

Figure 5: (a)Schematic view of the tractor with the spray boom. (b)Transformation from camera coordinate system to world coordinate system. 


\section{Results and discussion}

\subsection{Efficiency of the Gabor filter algorithm}

Some algorithms have been developed in our lab and have been tested on real data and in real in-field conditions but assessing and comparing them appeared difficult and uncertain[20, 17]. So we have developed a new and original method dedicated to site-specific weed management proposing to model photographs taken from a virtual camera placed in a virtual crop field with different common Weed Infestation Rates (WIR).

To assess the efficiency of this algorithm for crop row detection and crop/weed discrimination we created a dataset composed of 30 series of 17 images; for each series the initial weed infestation rate was fixed from $0 \%$ to $80 \%$ with a step equals to $5 \%$.

The comparison between the true $W I R_{\text {inter-row }}$ and the detected $W I R_{\text {inter-row }}$ demonstrates that the classification method leads to misclassification errors. To understand these errors (Fig.6.b) and to evaluate the accuracy of this method, we summarize the classification results in a confusion matrix which indicates the number of correctly and incorrectly classified pixels (both weed and crop classes). So the detected WI $R_{\text {inter-row }}$ is composed not only of weed correctly detected (WW) but also of crop incorrectly detected and assigned as weed (CW). The same is true for the detected Crop Rate. It is composed not only of crop correctly detected (CC) but also of weed incorrectly detected and assigned as crop (WC). Consequently, if CW $>$ WC it indicates that the algorithm of classification overestimates the weed detection and then detected $W I R_{\text {inter-row }}>$ initial $W I R_{\text {inter-row }}$. Concerning the detected $\mathrm{CR}$, if $\mathrm{WC}>\mathrm{CW}$ it indicates that the algorithm of classification overestimates the crop detection and so underestimates the weed detection. The figure, Fig.6.b, shows the results of the crop/weed discrimination with simulated images concerning either a punctual or a patchy spatial distribution for weeds in a crop field. For both cases (punctual and patchy distribution) the algorithm overestimates the crop detection and so underestimates the weed detection. Moreover, with the field modelling, we are able to highlight the limits of the efficiency of the algorithm for all values of inter-row WIR (real or unreal situations). In the case of high WIR (up to 40\%), the algortihm becomes inefficient. Fortunately, a real crop field with such a WIR does not exist.

(a)

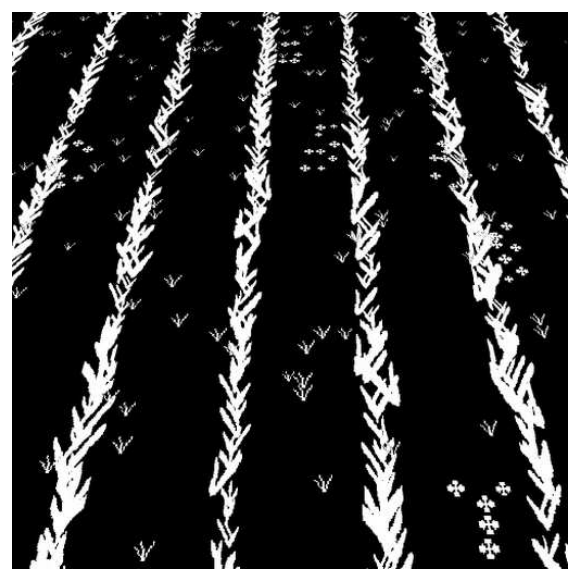

(b)

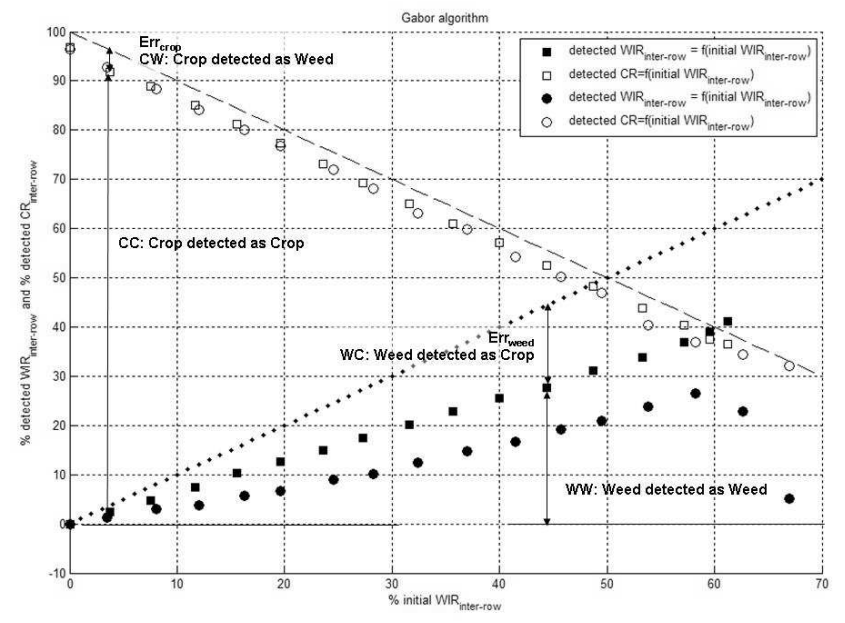

Figure 6: (a) virtual image of a wheat field with an initial inter-row WIR of 20\%. (b) Detected inter-row WIR and detected CR for a weed punctual distribution (square dot) and a weed patchy distribution (circle dot). 
Other crop/weed discrimination algorithms based on wavelet or Hough Transform, are currently tested owing virtual image. It should be noted that all these algorithms are enabled to estimate only an inter-row WIR. Consequently, if a weed is located in a crop row, it will not be detected. The accuracy of these algorithms is compared and it reveals that wavelets are well adapted for perspective images and provide better results than the Gabor filtering. However, the Gabor filtering has been implemented for a quick and easy development of the site-specific sprayer. Moreover Gabor filtering is rather adapted for real-time applications: easy implementation and short calculation time. The computing time of the treatment is less than one second implying a maximum tractor speed of $8.8 \mathrm{~km} / \mathrm{h}$. If we want to increase the speed of the tractor, we must decrease the computation time of our treatment and consequently we must use a more efficient processor.

\subsection{Preliminary tests}

At the moment, many different trials are realized indoors (Fig.3.a) or on artificial conditions in order to test the site specific spraying system. Although the results are quiet good (cf. video sample in additional file), the precision sprayer is efficient for a specific camera configuration and a specific cereal field. Now to optimize the spraying system we have developed calibration curves owing the vibrations of the tractor, the unevenness of the ground and the fact that the camera is inclined and not close to the sprayer boom. Indeed, the variations of the camera orientation induce a small shift $(\mathrm{few} \mathrm{cm})$ on the $x_{\text {weed }}$ and $y_{\text {weed }}$ positions in the field[21]. Consequently, to compensate for these error positions, we simply propose to add a delay on the EVP activation. This delay value will depend on the weed position in the perspective image. Concerning the image processing based on a Gabor filter, the parameters of the filter were equal to $u_{0}=0.0049, \sigma_{x}=108.65$ and $\sigma_{y}=32.60$ , these parameters were deduced from the initial image (Fig.3.a and Fig.3.b) with $f_{A}=0.0049, f_{B}=0.0029$, $f_{C}=0.0059$ and $f_{D}=-0.0020$. The detected inter-row Weed Infestation Rate (WIR) of the processed image (Fig.4.a) is $9.5 \%$.

\subsection{Validation tests}

We actually test the feasibility of the precision sprayer in real agronomic field. However, we are very dependent on the weather and the growth stage of the crop and so these experiences are more complicated to carry on. The experiences based on the plant/soil discrimination have been validated (cf. video sample in additional file) and the next experience concerns the crop/weed discrimination as soon as possible waiting for adequate crop growth-stage for an efficient herbicide treatment.

\subsection{Further research}

The improvements of the precision sprayer concern the image processing and particularly the crop/weed detection. Indeed the intra-row weed detection are not detected and so other image processing algorithms must be investigated. To improve our method of discrimination, it would be to interesting to combine this spatial information with spectral information. Indeed, these last decades, the spectral properties of the plant were studied for discrimination between crop and weeds[19]. Several methods based on reflectance of the plants also exist. Some use artificial networks[22, 23, 24, 25], others use the statistical analysis as the Principal Component Analysis (PCA)[26] or a Discriminating Factorial Analysis (DFA)[27]. Although the establishment of the discrimination between monocotyledon and dicotyledon based on spectral approach is realizable, the discrimination of the species has not clearly established. Indeed Bossu et al.[25] studied successfully such a discrimination under conditions of laboratory on leaves of various species of weeds, but they must confirm these results in real conditions. 


\section{Conclusion}

A machine vision system has been developed for a real time precision sprayer based on the image processing. The spatial method based on a Gabor filtering and a region-based segmentation, allows us to detect only interrow weeds. The precision sprayer has been tested only on a simulated agronomic scene. We are able to open the right EPV at the right moment and at the right place and the feasibility stage has been validated. Trials in real agronomic field are realized and are very promising. To improve the crop/weed detection and particulary the intra-row weed detection, other image processing algorithms must be investigated.

\section{Acknowledgments}

The authors thank Mr. M. Morel from Tecnoma company for sponsoring our research (http://www.tecnoma.com). We also thank R. Martin (technician), F. Voiry and A. Malashko (agroequipement students) for their help on the home-made sprayer.

\section{A Optical system}

In this part, we will present the optical transformation. Indeed, the camera is located with a height $H$ (millimeters) from the ground, and it is inclined with a tilted angle $\phi$ (degree) with the vertical as shows Fig.5.b. In order to determine the coordinates of a point in the real world $\left(x_{w}, y_{w}\right)$ from its coordinates in the image world $\left(x_{c}, y_{c}\right)$, we must characterize the matrix projection. The transformation of a position expressed in the camera coordinate system, $k$, to a position expressed in the world coordinate system, $w$, is given by:

$$
\left[\begin{array}{c}
x \\
y \\
w
\end{array}\right]^{w}=R_{k}^{w}\left[\begin{array}{l}
x \\
y \\
z
\end{array}\right]^{k}+\left[\begin{array}{c}
t_{x} \\
t_{y} \\
t_{z}
\end{array}\right]_{k_{\text {org }}}^{w}
$$

Where $R$ is the rotation matrix between real world system, $w$, and the camera system $k$. In our case, $R$ is function of $\phi$ :

$$
R_{k}^{w}=\left[\begin{array}{ccc}
1 & 0 & 0 \\
0 & \cos (\phi+180) & -\sin (\phi+180) \\
0 & \sin (\phi+180) & \cos (\phi+180)
\end{array}\right]
$$

In our case, the translation vector is a function of $H$ and $\phi$ :

$$
\begin{aligned}
t_{x} & =0 \\
t_{y} & =-H \tan (\phi) \\
t_{z} & =H
\end{aligned}
$$

So, the extrinsic parameter matrix is equal to:

$$
\left[\begin{array}{l}
x \\
y \\
z
\end{array}\right]^{w}=\left[\begin{array}{ccc}
1 & 0 & 0 \\
0 & -\cos \phi & \sin \phi \\
0 & -\sin \phi & -\cos \phi
\end{array}\right]\left[\begin{array}{l}
x \\
y \\
z
\end{array}\right]^{k}+\left[\begin{array}{c}
0 \\
-H \tan \phi \\
H
\end{array}\right]
$$

Moreover, to determine a position expressed in the camera coordinate system, $k$, the intrinsic parameters of the camera are required. We use the CCD image benchmark, $i$, where coordinates are in metric unit:

$$
\begin{aligned}
x_{k} & =\frac{x_{i}}{z_{i}} z_{k} \\
y_{k} & =\frac{y_{i}}{z_{i}} z_{k}
\end{aligned}
$$


The determination of the coordinates in the CCD image benchmark, $i$, are based on the coordinates expressed in pixels in the image benchmarks $c$ :

$$
\begin{aligned}
x_{i} & =\left(x_{c}-C_{x}\right) d_{x} \\
y_{i} & =\left(y_{c}-C_{y}\right) d_{y} \\
z_{i} & =f
\end{aligned}
$$

$f$ in millimeters corresponds to the focal length of the camera, and $C_{x}$ and $C_{y}$ are the coordinates of the optical center of the camera expressed in pixels, that corresponds to the half size of the image. $d_{x}$ and $d_{y}$ are the dimensions of a CCD element, horizontally and vertically respectively.

$$
\begin{aligned}
& x_{k}=\frac{\left(x_{c}-C_{x}\right) d_{x}}{f} z_{k} \\
& y_{k}=\frac{\left(y_{c}-C_{y}\right) d_{y}}{f} z_{k}
\end{aligned}
$$

If $s=\frac{z_{k}}{f} \Rightarrow z_{k}=f s$, then:

$$
\begin{aligned}
x_{k} & =x_{c} d_{x} s-C_{x} d_{x} s \\
y_{k} & =y_{c} d_{y} s-C_{y} d_{y} s \\
z_{k} & =f s
\end{aligned}
$$

So, the intrinsic parameter matrix is given by:

$$
\left[\begin{array}{l}
x \\
y \\
z
\end{array}\right]^{k}=\left[\begin{array}{ccc}
d_{x} & 0 & -C_{x} d_{x} \\
0 & d_{y} & -C_{y} d_{y} \\
0 & 0 & f
\end{array}\right]\left[\begin{array}{c}
s x \\
s y \\
s
\end{array}\right]^{c}
$$

If we use the homogeneous and uniform matrix, we can directly define the transformation of a position expressed in the image coordinate system, $c$, to a position expressed in the world coordinate system, $w$

$$
\left[\begin{array}{c}
k x \\
k y \\
k z \\
k
\end{array}\right]^{w}=\left[\begin{array}{cccc}
d_{x} & 0 & -C_{x} d_{x} & 0 \\
0 & -d_{y} \cos \phi & C_{y} d_{y} \cos \phi+f \sin \phi & -H \tan \phi \\
0 & -d_{y} \sin \phi & C_{y} d_{y} \sin \phi-f \cos \phi & H \\
0 & 0 & 0 & 1
\end{array}\right]\left[\begin{array}{c}
s x \\
s y \\
s \\
1
\end{array}\right]^{c}
$$

then :

$$
\begin{aligned}
& x_{w}=\frac{\left(x_{c}-C_{x}\right) d_{x} H}{\left(y_{c}-C_{y}\right) d_{y} \sin \phi+f \cos \phi} \\
& y_{w}=-\frac{2 H d_{y}\left(y_{c}-C_{y}\right)}{\left(y_{c}-C_{y}\right) d_{y} \sin (2 \phi)+f(1+\cos (2 \phi))} \\
& z_{w}=0
\end{aligned}
$$

\section{References}

[1] L. Tian, J.F. Reid, and J. Hummel. Development of a precision sprayer for site-specific weed management. Transactions of the ASAE, 42(4):893-900, 1999.

[2] J. B. Vioix. Conception et réalisation d'un dispositif d'imagerie multispectrale embarqué : du capteur aux traitements pour la détection d'adventices. PhD thesis, University of Burgundy, 2004. 
[3] R. Sugiura, N. Noguchi, and K. Ishii. Remote-sensing technology for vegetation monitoring using an unmanned helicopter. Biosystems Engineering, pages 369-379, 2005.

[4] Ntech Industries. http://www.ntecindustries.com.

[5] W. L. Felton and K. R. McCloy. Spot spraying. Agricultural Engineering, 11:26-29, 1992.

[6] W. L. Felton. Commercial progress in spot spraying weeds. In Brighton Crop Protection Conference Weed, British Crop Protection Council, volume 3, pages 1087-1096, Brighton (UK), 1995.

[7] B. Åstrand and A.-J Baerveldt. A mobile robot for mechanical weed control. International Sugar Journal, 105(1250):89-95, February 2003.

[8] B. Åstrand and A. J. Baerveldt. A vision based row-following system for agricultural field machinery. Mechatronics, 15:251-269, 2005.

[9] Intel. Open source computer vision library. http://www.intel.com/technology/computing/opencv/ index.htm.

[10] Intel. Intel integrated performance library. http://www.intel.com/cd/software/products/ asmo-na/eng/perflib/302910.htm.

[11] G. Jones, C. Gée, and F. Truchetet. Simulation of agonomic images for an automatic evaluation of crop/weed discrimination algorithm accuracy. In Eigth International Conference on Quality Control by Artificial Vision, volume 6356, pages 0J-1-0J-10, Le Creusot, France, May 2007.

[12] R. A. Fisher and R. E. Miles. The role of spatial pattern in the competition between crop plants and weeds. a theorical analysis. Mathematical Biosciences, 18:335-350, 1973.

[13] Y. Hamamoto, S. Uchimura, M. Watanabe, T. Yasuda, Y. Mitani, and S. Tomita. A gabor filter-based method for recognizing handwritten numerals. Pattern Recognition, 31:395-400, 1998.

[14] A. Jain and N. R. S. Lakshmanan. Object detection using gabor filters. Pattern Recognition, 30:295-309, 1997.

[15] J. Yang, L. Liu, T. Jian, and Y. Fan. A modified gabor filter design method for fingerprint image enhancement. Pattern Recognition Letters, 24:1805-1817, 2003.

[16] D. Gabor. Theory of communication. J. IEE (London), 93(III):429-457, November 1946.

[17] J. Bossu, C. Gée, J. P. Guillemin, and F. Truchetet. Development of methods based on double hough transform or gabor filtering to discriminate between crop and weed in agronomic images. In IS\&T/SPIE 18th Annual Symposium Electronic Imaging Science and Technology, volume 6070, pages 1-11, San Jose, California USA, January 2006.

[18] D. H. Ballard and C. M. Brown. Computer Vision. Prentice Hall, 1982.

[19] R. Deriche and J-P. Cocquerez. Extraction de composantes connexes basée sur une détection optimale des contours. In Cognitiva, pages 1-9. CESTA, 1987.

[20] J-B. Vioix, J-P. Douzals, F. Truchetet, L. Assemat, and J-P. Guillemin. Spatial and spectral methods for weed detection and localization. EURASIP Journal on Applied Signal Processing, 7:679-685, 2002.

[21] J. Bossu. Segmentation d'images pour la localisation d'adventices. Application à la rééalisation d'un systèème de vision pour une pulvéérisation spéécifique en temps rééel. (Image segmentation for weed detection. Development of a vision system for a real-time precision sprayer). $\mathrm{PhD}$ thesis, University of Burgundy (France), 2007. 
[22] D. Moshou, J. De Baerdemaeker, and H. Ramon. Neural network based classification of different weed species and crops. In J.V. Stafford, editor, Second European Conference on Precision Agriculture, pages 275-284, Odense, Denmark, 1999.

[23] D. Moshou, E. Vrindts, B. De Baerdemeker, and J. Ramon. A neural network based plant classifier. Computers and Electronics in Agriculture, 31(1):5-16, 2001.

[24] C. Gée, L. Bonvarlet, J. B. Magnin-Robert, and J. P. Guillemin. Weed discrimination by reflectance measurements using neural networks. In douzième colloque international sur la biologie des mauvaises herbes, 2004.

[25] J. Bossu, C. Gée, J. P. Guillemin, and F. Truchetet. Feasibility of a real-time weed detection system using spectral reflectance. In J.V. Stafford, editor, Fifth European Conference on Precision Agriculture, pages 123-130, Upsalla, Sweden, 2005.

[26] T. Borregaard, H. Nielsen, L. Nørgaard, and H. Have. Crop-weed discrimination by line imaging spectroscopy. Journal of Agricultural Engineering Research, pages 389-400, 1999.

[27] C. Gée, L. Bonvarlet, J.B. Magnin-Robert, and J.P. Guillemin. Weeds classification based on spectral properties. In $7^{\text {th }}$ International Conference on Precision Agriculture and Other Ressource Management, Minneapolis (USA), July 2004. 